\title{
Experimental Techniques to Study Physical, Electric, Dielectric and Magnetic Properties of Ferrites: A Survey
}

\author{
Suresh S. Shinde ${ }^{1}$ \\ Department of Physics, A.S.C. College, Naldurg, Osmanabad (M.S.) India ${ }^{1}$
}

\begin{abstract}
Various experimental techniques are used to study different properties of ferrites. Using X-ray diffraction, physical and structural properties like lattice constant, X-ray density, porosity, particle size, theoretical lattice constant and cation distribution are determined. IR study is useful to determine position of different vibration modes, bond lengths and force constant. Under electric and dielectric study, dc resistivity, ac resistivity, dielectric constant, dielectric loss and dielectric loss tangent are determined. Magnetic properties are measured using pulse field magnetic hysteresis loop tracer. Under this study magnetic properties like saturation magnetization, coercivity, remanence magnetization etc are measured. Present paper reports use of these experimental techniques to study various properties of ferrites.
\end{abstract}

Keywords: Lattice constant, porosity, dielectric constant, hysteresis, magnetization.

\section{INTRODUCTION}

Ferrites are magnetic ceramics usually composed of oxides of iron and other materials possessing combined properties of magnetic conductor and electrical insulator. The increasing demand for low loss ferrites resulted in detailed investigations on the various aspects of the conductivity and effect of various substitutions on the electrical conductivity, thermoelectric power, hall mobility and magnetic properties.

Magnetic oxides are used in many technological applications such as permanent magnets, microwave absorbers, chemical sensors[1,2]. Their magnetic and electrical properties depend on several factors such as method of preparation, chemical composition, sintering temperature, sintering atmosphere and distribution of cations at tetrahedral (A-site) and octahedral [B-site] sites [3]. Spinel ferrites are very sensitive to the manufacturing process. Soft ferrite crystallizes in spinel structure in which cations can be found in tetrahedral (A) and octahedral [B] site. The methods of preparation plays an important role in governing the basic properties of ferrite. Spinel ferrites are usually prepared by ceramic technique.

The substitution of non-magnetic ions produces variations in the properties of spinel ferrite. The structural, electrical and magnetic properties of ferrite system are investigated by means of X-ray diffraction, infrared spectroscopy, d.c. resistivity a.c. resistivity, magnetization and a.c. susceptibility measurements.

\section{Structural Properties by X-Ray Diffraction}

The powdered X-ray diffraction patterns of the ferrite samples are recorded at room temperature using $\mathrm{Cu}-\mathrm{K} \alpha$ radiation on Philips (PW3710) X-ray diffractometer. X-ray diffraction data is collected in the $2 \theta$ range of $20^{\circ}$ to $80^{\circ}$ with scanning rate $2^{0}$ minute ${ }^{-1}$. The X-ray diffraction showed sharp Bragg peaks corresponding to single phase spinel. The XRD data is used to determine the values of lattice parameter, X-ray density, particle size and cation distribution.

Following formulas are used for calculation of various parameters,

\section{Lattice constant}

The lattice parameter ' $\mathrm{a}$ ' is calculated from the inter-planer spacing values using the following equation for cubic structure [6]

$\mathrm{a}=d \sqrt{\left(h^{2}+k^{2}+l^{2}\right)}$

Bulk density [7] is calculated by considering the cylindrical shape of the pellets and using the relation

$d_{B}=\frac{m}{V}=\frac{m}{\pi r^{2} h}$

Where $\mathrm{m}$ is the mass, $\mathrm{r}$ is radius and $\mathrm{h}$ is thickness of the pellet

$X$-ray density

X-ray density $d_{\mathrm{X}}$, is calculated using the expression [8] 
$d_{x}=\frac{8 M}{N_{A} a^{3}}$

where ' $\mathrm{N}_{\mathrm{A}}$ ' is the Avogadro's number and ' $\mathrm{a}$ ' is the lattice constant and $\mathrm{M}$ is the molecular weight of the composition.

\section{Porosity}

The porosity is calculated using the relation [9]

$d_{x}=\left(1-\frac{d_{B}}{d_{X}}\right)$

where $d_{B}$ is the bulk density

\section{Crystallite size}

The average crystallite sizes were calculated from the X-ray diffraction peaks using Scherer's formula [10]

$t=\frac{k \lambda}{B \cos \theta}$

where $\mathrm{t}$ is the crystallite size, $\lambda$ the $\mathrm{X}$-ray wave length $(=1.5405 \AA), \mathrm{k}$ is a constant $(=0.94), \mathrm{B}$ the broadening of the diffraction peak and $\theta$ is the diffraction angle.

\section{Theoretical lattice constant}

Theoretical lattice constant is calculated using following relation,

$\mathrm{a}_{\mathrm{th}}=\left(\frac{8}{3 \sqrt{3}}\right)\left[r_{A}+R_{0}\right]+\sqrt{3}\left[r_{B}+R_{0}\right]$

Where $r_{A}$ and $r_{B}$ are the site radii of tetrahedral $A$ and octahedral $B$ site respectively and $R_{0}$ is the radius of oxygen ion.

\section{Cation distribution}

The cation distribution is obtained from the analysis of intensity of X-ray diffraction patterns. In this method the observed intensity ratios are compared with the calculated intensity ratios. Buerger method [7 - 11] is used to determine the cation distribution. This method selects a few pairs of reflections according to the expression

$$
\frac{\mathrm{I}_{\mathrm{hkl}}^{\mathrm{Obs}}}{\mathrm{I}_{\mathrm{h}^{\prime} \mathrm{k}^{\prime} \mathrm{l}^{\prime}}^{\mathrm{obs}}}=\frac{\mathrm{I}_{\mathrm{hkl}}^{\mathrm{Cal}}}{\mathrm{I}_{\mathrm{h}^{\prime} \mathrm{k}^{\prime} \mathrm{l}^{\prime}}^{\mathrm{Cal}}}
$$

where, $\mathrm{I}_{\mathrm{hkl}}^{\text {Obs. }}$ and $\mathrm{I}_{\mathrm{hkl}}^{\text {Calc. }}$ are observed and calculated intensities respectively. The best information on cation distribution is achieved when comparing experimental and calculated intensity ratios for reflections whose intensities (i) are nearly independent of the oxygen parameter, (ii) vary with the cation distribution in opposite ways and (iii) do not differ significantly.

Selected planes are used to calculate intensity ratio which are assumed to be sensitive to the cation distribution. The temperature and absorption factors are not considered in the calculations as they do not affect the intensity calculation. If an agreement factor ( $\mathrm{R}$ ) is defined as in following equation then the best-simulated structure which matches the actual structure of the sample will lead to a minimum value of $\mathrm{R}$ and the corresponding cation distribution is obtained for each hkl and $h^{\prime} k^{\prime} l^{\prime}$ reflection pair considered.

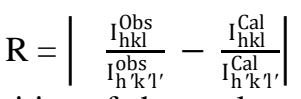

The intensities of these planes are nearly independent of the oxygen parameters. The calculations were made for various combinations of cations. To calculate the relative integrated intensity of a given diffraction line from powder specimens as observed in a diffractometer with a flat-plate sample holder, the following formula is valid as suggested by Buerger [11]

$$
\boldsymbol{I}_{h k l}=|\boldsymbol{F}|_{h k l}^{2} \boldsymbol{P} \cdot \boldsymbol{L}_{P}
$$

where, $\mathrm{F}$ is structure factor, $\mathrm{P}$ is multiplicity factor, $\mathrm{L}_{\mathrm{P}}$ the Lorentz polarization factor where

$$
L_{P}=\frac{1+\cos ^{2} 2 \theta}{\sin ^{2} \cos ^{2} 2 \theta}
$$

The atomic scattering factor for various ions was taken from the literature [7 ]. 


\section{Infra Red Spectroscopy (IR)}

To record IR spectra of the ferrite system, the powdered samples are mixed with $\mathrm{KBr}$ in the ratio 1:250 by weight. Then the mixed powder is pressed in cylindrical die to obtain pellets of $1 \mathrm{~mm}$ thickness. The IR spectra is recorded in the range of $200-800 \mathrm{~cm}^{-1}$ at room temperature on the infrared spectrometer. The position of different vibration modes have been noted from these spectra. The IR spectra are used to determine bond length $R_{A}$ and $R_{B}$, in a cubic crystal for tetrahedral (A) and octahedral [B] site using formula given by Gorter [13]. Using the analysis of Waldron [14], the force constant $K_{0}$ and $K_{t}$ were calculated by using molecular weights of cations at tetrahedral (A) and octahedral [B] sites and absorption wave number $v_{1}$ and $v_{2}$.

$$
\begin{gathered}
\mathrm{R}_{\mathrm{A}}=\left(u-\frac{1}{4}\right) a \sqrt{3}-r\left(O^{-2}\right) \\
\mathrm{R}_{\mathrm{B}}=\left(\frac{5}{8}-u\right) a-r\left(O^{-2}\right)
\end{gathered}
$$

\section{Force Constant}

The force constants corresponding to the tetrahedral and octahedral complexes are calculated by using the standard formulae given below,

$$
\begin{aligned}
& K_{t}=7.62 \times M_{1} \times v_{1}^{2} \times 10^{-7} \\
& K_{O}=10.62 \times \frac{M_{2}}{2} \times v_{2}^{2} \times 10^{-7}
\end{aligned}
$$

where $K_{O}$ is the force constant on octahedral site, $K_{t}$ is the force constant on tetrahedral site, $M_{1}$ is molecular weight of A-site, $M_{2}$ is molecular weight of B- site, $v_{1}$ is the corresponding center frequency on tetrahedral site, and $v_{2}$ is the corresponding center frequency on octahedral site. The molecular weights $M_{1}$ and $M_{2}$ for each sample are calculated from the cation distribution.

\section{D.C. Resistivity}

The d.c. electrical resistivity of all the samples as a function of temperature were measured by two probe technique. The measurements were recorded in the temperature range of $300 \mathrm{~K}$ to $800 \mathrm{~K}$. The typical sample holder has been designed for measuring the electrical resistivity. Each sample was held in furnace. The temperature of each sample was measured using chromel-alumel thermocouple with an accuracy $\pm 5 \mathrm{~K}$. The resistance of pellet has been measured with rising and falling temperatures in the step of $5 \mathrm{~K}$. During each measurement, sufficient time was allowed for the sample to attain the equilibrium temperature. Finally, the resistivity of all the samples was calculated using following formula.

$$
\rho=\frac{\boldsymbol{R} A}{t}
$$

where, $\mathrm{R}$ is resistance of pellet, $\mathrm{A}$ area of pellet, and $\mathrm{t}$ is thickness of pellet.

\section{Dielectric constant}

The dielectric constant $\left(\varepsilon^{\prime}\right)$ is calculated using the following relation

$$
€^{\prime}=\frac{\mathrm{C}_{\mathrm{p}} \mathrm{d}}{€_{0} \mathrm{~A}}
$$

where, $\mathrm{Cp}$ is capacitance measured, $\mathrm{d}$ is thickness of pellet, $\varepsilon_{0}$ is permittivity of the free space, A is cross sectional area of pellet. The dielectric loss $\left(\varepsilon^{\prime \prime}\right)$ was calculated using the relation ,

$$
€^{\prime \prime}=\tan \delta €^{\prime}
$$

The values of dielectric loss tangent $(\tan \delta)$ were calculated using the relation

$$
\tan \delta=\frac{\varepsilon^{\prime \prime}}{\varepsilon^{\prime}}
$$

\section{Magnetization measurements:}

\section{Magnetic hysteresis loop tracer:-}

Magnetic properties such as saturation magnetization, coercivity, remanence magnetization etc. are measured using pulse field magnetic hysteresis loop tracer. The measurements were carried out at room temperature. The system (fig.1) consists of pulse power supply, solenoid and pick up coil assembly, signal processor and data acquisition system. 


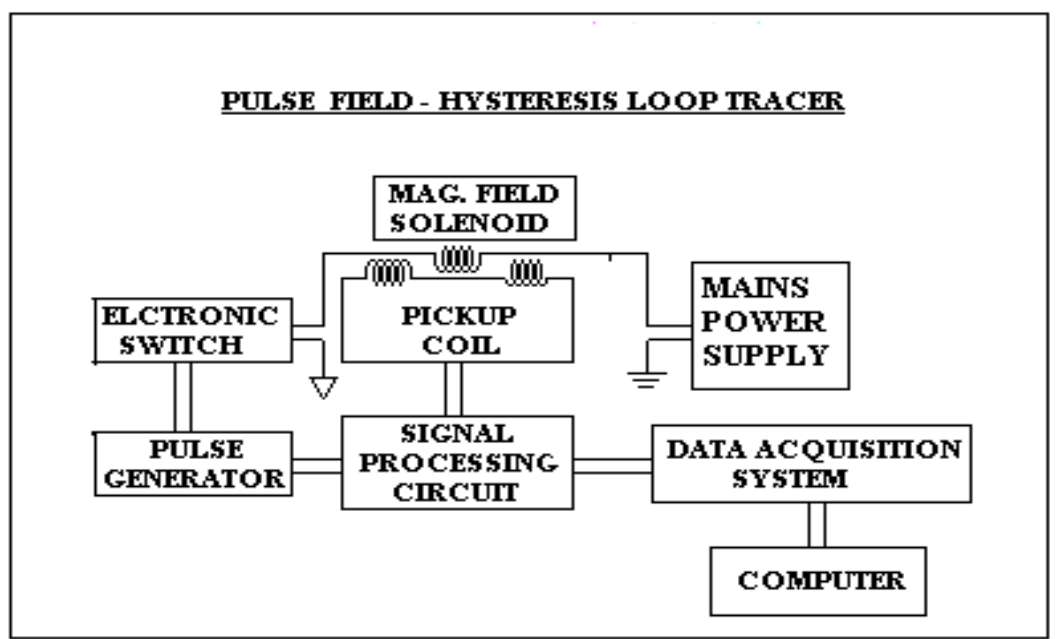

Fig.1: Schematic diagram of pulse field hysteresis loop tracer system

\section{Solenoid and Pick-up Coil Assembly:}

The solenoid is wound on a high quality Bakelite former with an enameled copper wire. A pickup coil system is wound with a fine copper wire on a former concentric with the solenoid. It consists of two main windings whose resultant voltage is very nearly balanced. An additional winding is used for perfect balance. The output of this winding is connected to a balancing network consisting of a magnitude and phase balance. The assembly containing the solenoid and pickup coil is placed in a acrylic box.

\section{Signal Processor:}

The signals produced in the pickup coil are proportional to the rate of change of magnetization of the sample as well as to the applied field. These are processed using integrating, amplifying and phase correcting circuits to produce signals representatives of magnetization of the sample and the applied magnetic field.

\section{Data Acquisition System (DAS):}

The Data Acquisition System consists of a micro-controller with fast digitizing and high resolution circuit. The digitized data is sent to a PC through RS-232 port. Special software is designed to plot the hysteresis loop and display the hysteresis parameters.

Measurements are taken after applying magnetic field of 5 KOe having the magnetization sensitivity 10 emu with accuracy 1 to $5 \%$. High magnetic field is generated in a solenoid by passing a pulse current of sinusoidal shape. A pickup coil system is kept in solenoid to detect field and magnetization signal of a sample in the pickup coil. The signals produced are then processed by an electronic system. These processed signals are digitized by a microcontroller and then sent to computer for plotting a hysteresis loop. Hysteresis loop is observed on the monitor with calculated values of hysteresis parameters. The hysteresis loop gives the values of coercivity $\left(\mathrm{H}_{\mathrm{c}}\right)$, remnant magnetization $\left(\mathrm{M}_{\mathrm{r}}\right)$ and saturation magnetization $\left(\mathrm{M}_{\mathrm{s}}\right)$.

\section{$6 \quad$ A.C. Susceptibility measurements:}

The Curie temperature of each sample was determined from the low field a.c. susceptibility data. The measurements of a.c. susceptibility are carried out in the temperature range of $300-800 \mathrm{~K}$. From the plots of $\chi_{\mathrm{T}} / \chi_{\mathrm{RT}}$ versus temperature, Curie temperature of the sample is obtained.

The Curie temperature setup consists of

1 A.C. susceptibility unit,

2 Solenoid and pickup coil assembly,

3 Platinum furnace,

$4 \quad$ Furnace power supply,

5) Thermocouple and temperature indicator.

The a.c. susceptibility measurements of the powdered samples are measured using the set up developed by Likhite et. al. shown in Fig.2. It consists of Helmholtz coil, two pick-up coils, furnace and sample holder. The set up operates at frequency of $263 \mathrm{~Hz}$ and in the r.m.s. field of 7 Oe.

The set up consist of two Helmholtz coil to produce uniform field at pick up coil. To heat the sample from room temperature to about $800 \mathrm{~K}$, a furnace was fabricated by winding the platinum wire on silica tube. To avoid overheating of coil a glass jacket with water circulation was used. The furnace was inserted in the glass jacket and was placed at the center of pick up coil. The sample holder was made up of a quartz tube fused at one end. The height of the sample holder was maintained such a way that the sample can stay at center of coils. The current was supplied to the Helmholtz 
coil by power supply and induced emf was displayed on a digital voltmeter. The signal induced in the double coil, which is proportional to the rate of change of magnetic moment of the specimen, is amplified, rectified and read out on digital voltmeter. The temperature of furnace was measured by using Platinum-Platinum-Rhodium thermocouple. The powdered sample was gradually heated and at various temperatures, signals corresponding to the magnetic moment were taken. The measurements were taken from room temperature up to Curie temperature $\mathrm{T}_{\mathrm{C}}$

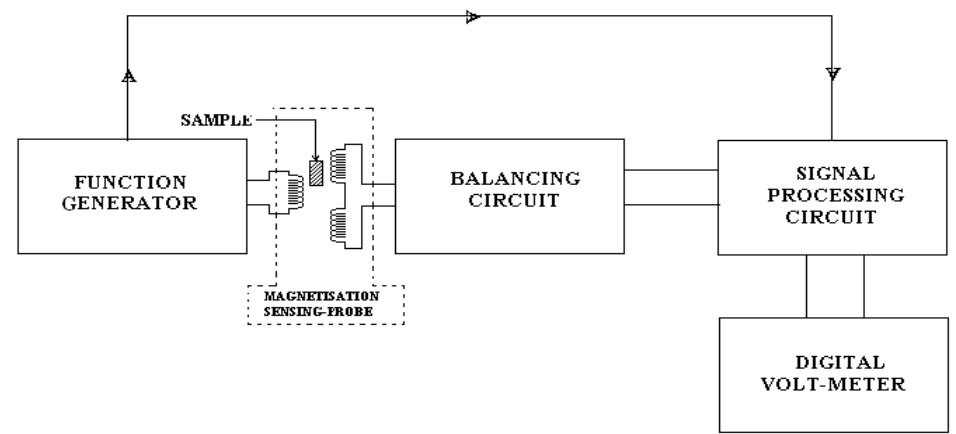

Fig. 2 Schematic of a.c. susceptibility system

\section{Bohr Magneton}

The saturation magnetization, coercivity, remanence magnetization etc. magnetic properties are measured using pulse field magnetic hysteresis loop tracer supplied by Magenta Company (Mumbai). The measurements were carried out at room temperature.

The magnetic moment per formula unit in Bohr magneton $\left(\mathrm{n}_{\mathrm{B}}\right)$ is calculated by using the relation,

$$
n_{B}=\frac{M_{W} \times M_{S}}{5585}
$$

where $M_{W}$ is molecular weight of the composition and $M_{S}$ is saturation magnetization.

The A.C.Susceptibility measurements on powdered samples are made in the temperature range 300 to $850 \mathrm{~K}$ using double coil setup operating at frequency of $263 \mathrm{~Hz}$ and in r.m.s. field of $39.8 \mathrm{Am}^{-1}$.

\section{CONCLUSION}

In this paper different experimental techniques are explained in detail how they are used to study different properties of ferrites. Using these techniques, physical and structural properties like lattice constant, X-ray density, porosity, particle size, theoretical lattice constant and cation distribution ferrites are determined using X- ray diffraction . Position of different vibration modes, bond lengths and force constant are determined by IR study.. DC resistivity, ac resistivity, dielectric constant, dielectric loss and dielectric loss tangent are determined under electrical properties. Magnetic properties are measured using pulse field magnetic hysteresis loop tracer.

\section{REFERENCES}

[1] More R.M., Shinde T. J., Chaudhari N. D. and Vasambekar P. N. J. Mat. Sci. Mat. In. Electronics 16 (2005) 721.

[2] Stoppels D., J. Mag. Mag. Mat. 160 (1996) 323.

[3] Taylor J.A., Reczek S.T. and Rosen, Am. Ceram. Soc. Bull. 74 (1995) 91.

[4] Gul I.H., Ahmed W., Maqsood A., J. Mag. Mag. Mat. 320 (2008)270-275.

[5] Amanullah Choudhury M., Rahman J. J. Mag. Mag. Mat. 223(2001)21-26.

[6] Abo El Ata A.M., Attia S.M., El Kony D., Al-Hammadi A.H. J. of Magnetism and Magnetic Materials 295 (2005) 28

[7] George M, Nair S S, Malini K A, Joy P A Anantharaman M R J. Phys. D: Appl. Phys. 40 (2007) 1593

[8] Abdeen A.M., Hemeda O.M., Assem E.E., El-Sehly M.M. J. of Magnetism and Magnetic Materials 238 (2002) 75

[9] Hossaina A.K.M.A, Biswas T.S., Mahmud S.T., Yanagida T., Tanakab H., Mater Chem and Physics Xxx (2008) xxx

[10] Zheng Z.G., Zhong X.C., Y.H. Zhang, Yu H.Y., Zeng D.C. J. of Alloys and Compounds 466 (2008) 377

[11] Buerger M.J. "Crystal Structure Analysis", John Willey,New York (1960)

[12] Amer M.A., Hiti M. El J. of Magnetism and Magnetic Materials 234 (2001) 118

[13] Gortor E.W. Philips Res. Rep, 9 (1954) 295

[14] Waldron R.D. Phys. Rev. 99 (1955) 1707 